\title{
Development of data acquisition system for digital display and measurement tools based on Bluetooth SONG Ying ${ }^{1, a}$, Cheng Shuang ${ }^{2, b}$ and Feng Xuezhi, ${ }^{3, c}$
}

\author{
${ }^{1}$ Beijing Precision Engineering Institute for Aircraft Industry, china \\ ${ }^{2}$ Beijing Precision Engineering Institute for Aircraft Industry, china \\ ${ }^{3}$ Beijing Precision Engineering Institute for Aircraft Industry, china \\ ayingsong_8@hotmail.com, b chengshuangcs823@163.com, 675454128@qq.com
}

\begin{abstract}
Keywords: Interface Forms; Bluetooth; Bluetooth information
Abstract. In view of the fast and effective quality inspection requirement of IOT technology, based on the inherent communication protocol and electrical interface of the gage, we develop the digital measuring tool networking and data acquisition technology based on wireless technology, and integrate data acquisition of different digital display tools by Bluetooth technology. Finally, accurate uploading of measurement data has been realized, and the reliability and validity of the method are verified.
\end{abstract}

\section{Introduction}

Digital measurement technology has become an indispensable part of digital manufacturing technology. In recent years, digital measuring instruments and digital measuring instruments have developed rapidly. Digital display in various manufacturing industries, especially in traditional machinery manufacturing industry, is taking the strong momentum to replace traditional measuring tools, becoming the leading measuring instrument in the field of production

Automatic data acquisition of universal measuring tools is a key problem in the implementation of digital detection technology. At present, the measuring appliance manufacturers have launched a wireless digital display measuring output function, however, digital display measuring measuring instrument of different manufacturers have different wireless transmission protocol, the corresponding installation of different software or software corresponding to the same brand of different kinds of measuring tools, the need to manually configure the data line name and other information can realize the acquisition, this inconvenience for the automatic data collection instruments, the technical scheme of unified interface, the host computer for measuring with the uniform interface. The networking and data acquisition technology of digital measuring tools based on wireless technology will become the necessary technical means to improve the level of detection efficiency and quality management. [1].

Overall development scheme. Based on the measurement request, select the appropriate measurement tool to measure, obtain the corresponding measurement results; the measurements of signal conversion and tools and the measuring gauge identification, the identification of the articles measuring measurement data and storing information; the measuring mark will bring the measurement data information convert Bluetooth standard communication and data storage; the Bluetooth communication data into PC through the host computer interface circuit signal and transmits to the host computer and stored; the measuring device is identified by the host computer, the query and access articles identified the gage measuring information, will the measurement results of additional measuring information packet is sent to the measurement request. 


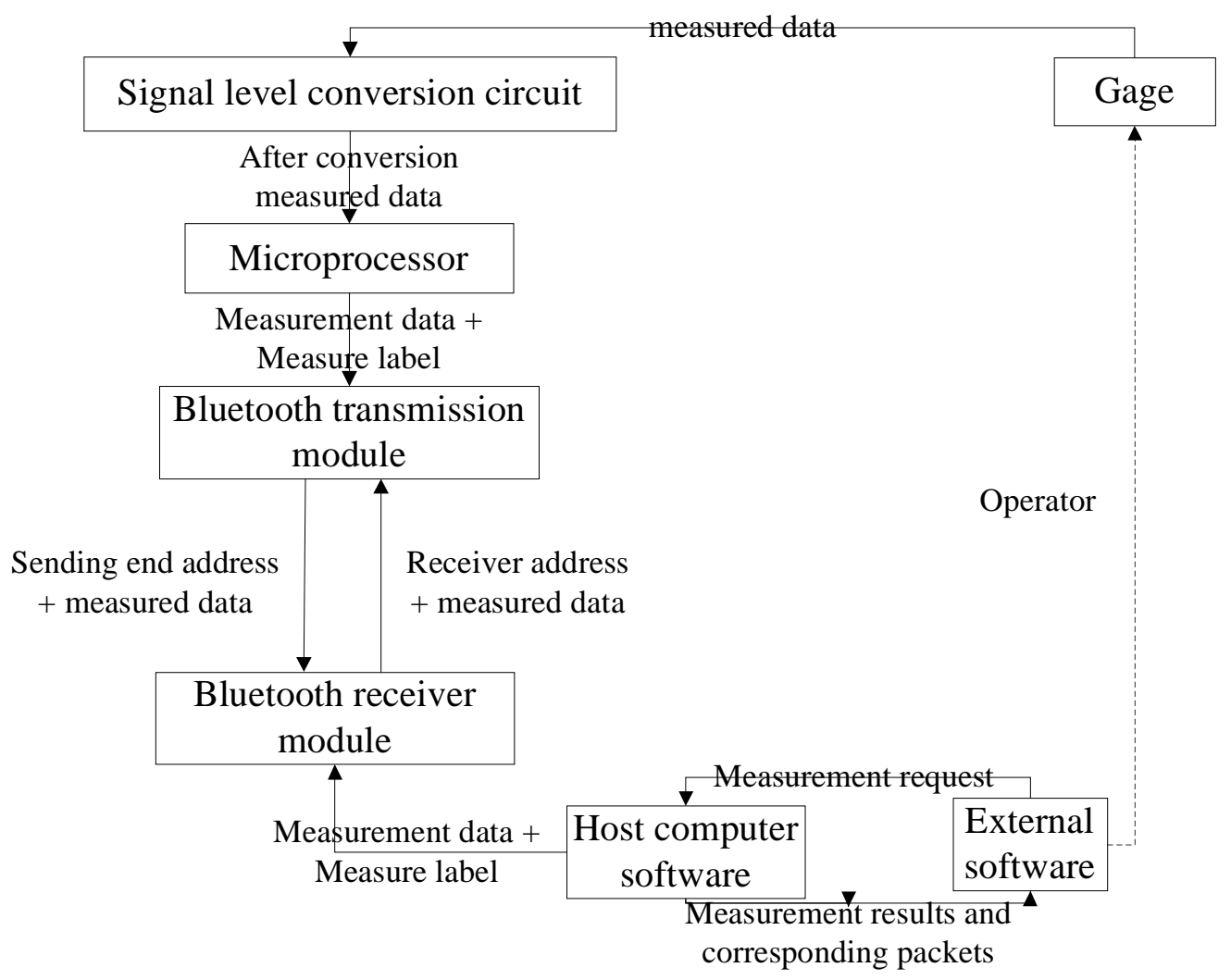

Fig. 1 system structure

Hardware development. The production site inspection data is dispersed in factories everywhere, such as: measuring machine, caliper, roughness instrument, image measurement equipment, these devices generate data have their own format, through their software to translate, if all direct collection of data in a database, you need to these data will be converted into the same format after storage, the hardware and software needs a unified interface. According to the difference of user's hardware configuration, the interface types of digital display gauge are also different, such as R S232 interface, USB interface, wireless interface and other interfaces with special functions.

Among them, the measurement module is configured to be based on the measurement request, and the corresponding measurement tool is selected to measure the corresponding measurement results. The measuring module can be set on the measuring tool. After sending the measurement request to the upper computer software program through external software, the corresponding measuring tool is selected to get the measurement result of the object to be measured.

The data processing module is configured for the measurement results for the signal conversion processing and measuring tools measuring tool identification, synthesis with measurement data storage and measuring identification, data processing module is provided with signal conversion and data processing function of the microprocessor, the invention is preferably used is Silicon Laboratories's chips C8051F340.

The Bluetooth communication module is configured to convert the measured data information with the measure label into the Bluetooth standard communication data and store it. The Bluetooth communication module includes Bluetooth sending unit and Bluetooth receiving unit, and the Bluetooth sending unit is configured to send the Bluetooth standard communication data to the Bluetooth receiving unit. And the Bluetooth communication module can be mounted on the storage medium, can be a memory with a Bluetooth communication protocol, data transmission unit through the Bluetooth Bluetooth receiver from the processing module and the data processing by the Bluetooth protocol to the Bluetooth receiving unit in the Bluetooth receiver unit side general data transmission interface.

The data upload module is configured to convert the Bluetooth standard communication data into the upper computer's circuit signal through the interface and upload it to the upper computer. The PC 
interface has a USB interface, and the interface of the digital display measure has the RS232 interface, the RS485 interface, the Opto232 interface and / or the external wireless interface.

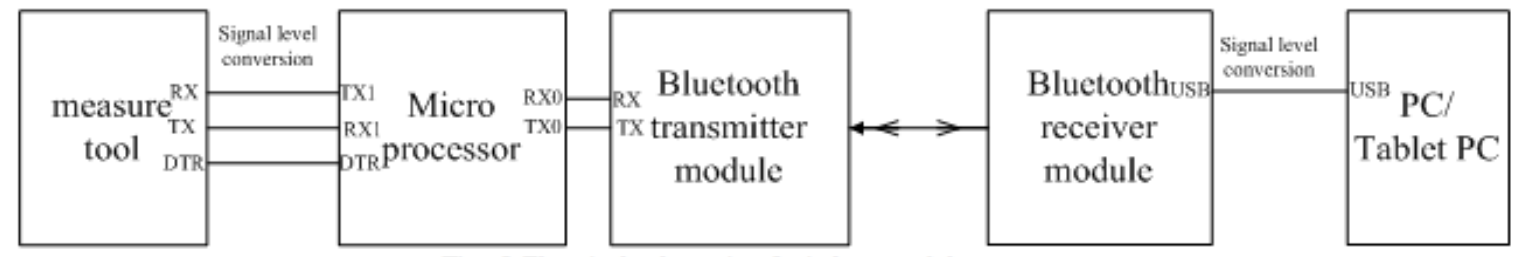

Fig. 2 Electrical schematic of wireless module

software development. Through the upper computer in the digital display system, the external measurement request is received, and the receiving measurement result data state is opened at the same time. At this point, the operator can choose the measuring tool adapted to the measurement request to measure the measured object and obtain the corresponding measurement results. Methods of measurement tools obtained through signal level conversion circuit for converting signal, and the processing method for measuring signal conversion results through the microprocessor to match additional measuring identification, synthesis with storage measurement data measuring mark, then carrying the measurement data is converted to the Bluetooth standard gauge identification communication and data storage and transmission.

Through the upper computer in the digital display system, the external measurement request is received, and the receiving measurement result data state is opened at the same time. At this point, the operator can choose the measuring tool adapted to the measurement request to measure the measured object and obtain the corresponding measurement results. Methods of measurement tools obtained through signal level conversion circuit for converting signal, and the processing method for measuring signal conversion results through the microprocessor to match additional measuring identification, synthesis with storage measurement data measuring mark, then carrying the measurement data is converted to the Bluetooth standard gauge identification communication and data storage and transmission.

The Bluetooth standard communication protocol to send data via Bluetooth and Bluetooth data stored in the module, through the use of Bluetooth protocol, capable of converting digital display measuring different manufacturers of different brands of data for the Bluetooth standard unified communication data, and then through the Bluetooth protocol mode will bring the measuring result of the measuring data and digital display measuring identification information transmitted and stored in the the corresponding storage carrier, connected by a system storage carrier and PC PC terminal or tablet computer, to achieve a unified data conversion and upload.

\section{Conclusions}

The measurement results will be the data measurement tools for signal conversion and additional measuring identification, and then carried into the Bluetooth standard communication data signal and Bluetooth protocol for wireless transmission in the storage medium, connected through the storage medium and the target device interface, can carry a way of measuring results measuring identification data information to point to specific equipment for transmission. The transmission protocol based on Bluetooth technology for unified conversion processing, data acquisition can be applied to different digital measuring tool, with measurement results of measuring data identified the measurement results with reliable traceability, to further improve the accuracy of measurement data. Therefore, the method and system of the invention have high integration, simple operation, accurate data and traceability.

\section{Acknowledgements}

This work was financially supported by the Technical Foundation (JSZL2015205A005). 


\section{References}

[1] X.C. Ming: Discussion on the type and development of digital display gauge interface. Low Carbon World. Vol. 12 (2014), p. 2

[2] Z.H.Long, L.Zhi: A data acquisition device for digital display and measure based on USB . , 201420303201.4, China, 2014 